\title{
MASS LOSS FROM CORONAE AND ITS EFFECT UPON STELLAR ROTATION
}

\section{KYOJI NARIAI}

\section{GPO PRICE S}

CSFTI PRICE(S) S

Hard copy (HC)

Microfiche (MF)

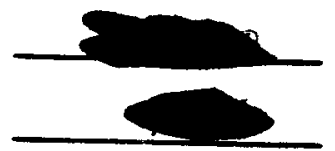

ff 653 July 65

SEPTEMBER 1968
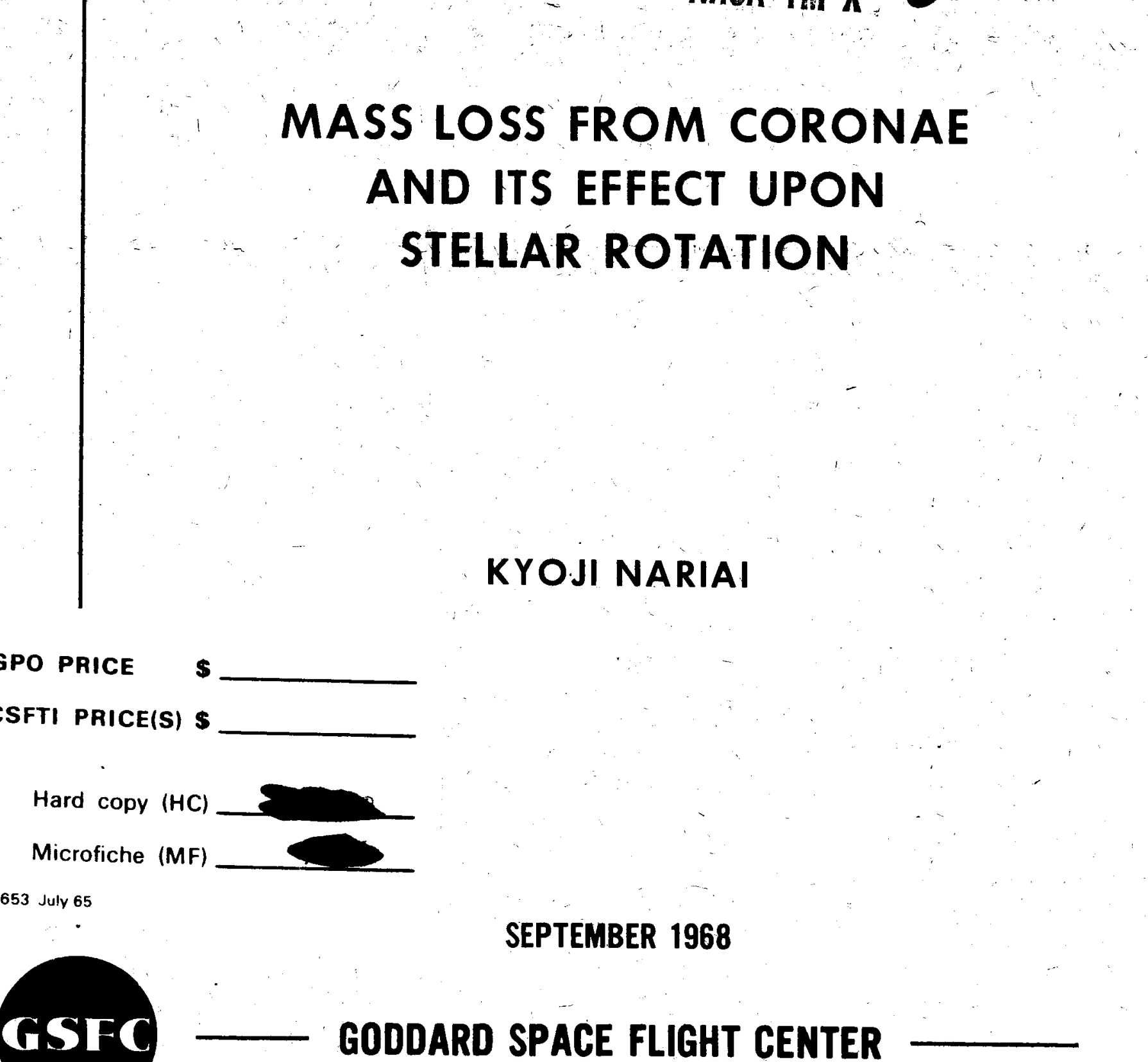
GREENBELT, MARYLAND
N $68-35731$

8
GODDARD SPACE FLIGHT CENTER

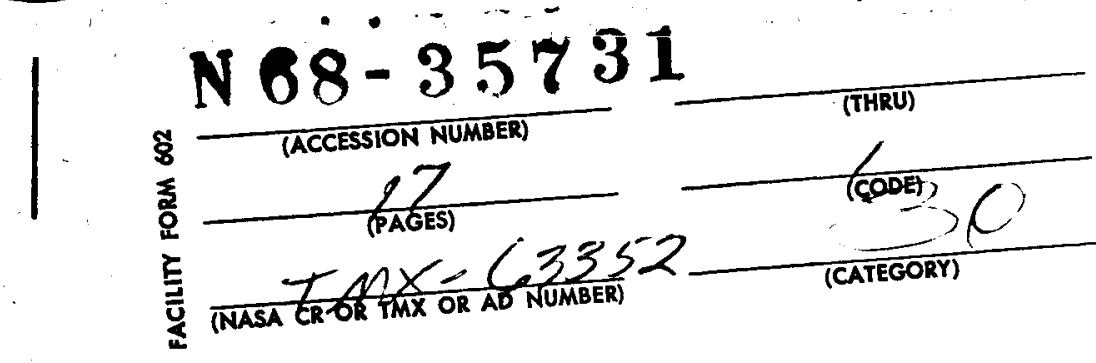

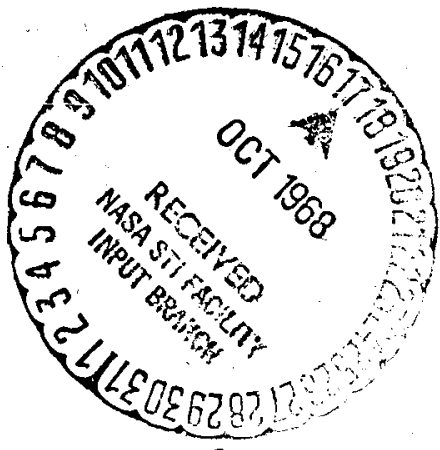




\title{
MASS LOSS FROM CORONAE AND ITS EFFECT UPON STELLAR ROTATION
}

\author{
Kyoji Nariai
}

September 1968

NASA-Goddard Space Flight Center

Greenbelt, Maryland, U.S.A. 


\title{
$\zeta$ \\ PRECEDING PAGE/BLANK NOT FILMED.
}

\author{
MASS LOSS FROM CORONAE AND ITS EFFECT \\ UPON STELLAR ROTATION
}

Kyoji Nariai*

\begin{abstract}
The acoustic energy generation rate from the convective zone was calculated for various models. Results show that chromosphere and corona can be expected around stars with temperature lower than $8,000{ }^{\circ} \mathrm{K}$ at the main sequence, and lower than $6,500^{\circ} \mathrm{K}$ at $\log \mathrm{g}=2$.

When a star is rotating rapidly, mass loss from its corona is large, and can be an effective mechanism of braking the stellar rotation. If this mechanism is effective, we can explain the slow rotation of stars later than F2 to be the result of the loss of the angular momentum through a stellar wind that is effective in their main sequence phase. Stars with mass $M>1.5 \mathrm{M}_{\odot}$ lose mass through a stellar wind during their contraction phase. The mass loss rate is larger than the solar value because of the larger energy input into the chromosphere-corona system and because of the smaller gravitational potential at the surface. $T$ Tauri stars may be the observational counterparts for such stars. As the duration of contraction phase is very short (less than $10^{7}$ years), the braking mechanism works only in the presence of a strong magnetic field (Ap) or in the presence of a companion (Am).
\end{abstract}

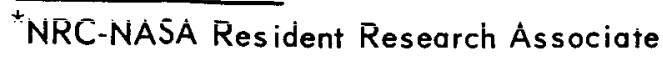




\section{MASS LOSS FROM CORONAE AND ITS EFFECT}

UPON STELLAR ROTATION

\section{ENERGY SUPPLY TO THE STELLAR CORONA}

Chromospheres and coronae are characterized by their high values of the ratio of the electron temperature to the radiation temperature and by their small optical depths, both measures being extreme in the coronae. Here the radiative process works only as a cooling mechanism. The dissipation of mechanical energy suggested by Biermann (1948), Schwarzschild (1948), and Schatzman (1949) seems to be adequate for heating the chromosphere and the corona. The rate of the acoustic energy generation in the solar convection zone with Lighthill's (1952) equation gives roughly the same value as the rate of energy loss from the solar chromosphere and corona by conduction and radiation (Osterbrock 1961). Therefore we may say that a star has a corona if the turbulent motions in the convective region are violent enough to produce the mechanical energy required to maintain the chromosphere-corona.

\section{ATMOSPHERIC MODELS}

In order to see the dependence of the convective zone on $\mathrm{Te}$ and $\mathrm{g}$, we have calculated a grid of model atmospheres with Böhm-Vitense's (1958) mixing-length theory as modified by Henyey, Vardya, and Bodenheimer (1965) who took into account Spiegel's (1957) suggestion concerning the temperature distribution in the turbulent element.

A stellar atmosphere is convectively stable when

$$
\left(\frac{d \ln T}{d \ln p}\right)_{a d}>\left(\frac{d \ln T}{d \ln p}\right)_{r a d}
$$

Near the surface, the atmosphere is stable and is in radiative equilibrium. The temperature and pressure distribution can be obtained by integrating the hydrostatic equation

$$
\mathrm{dp}=-\rho \mathrm{gdh}=\frac{\mathrm{g}}{\bar{K}} \mathrm{~d} \bar{\tau}
$$


with the help of the temperature dependence on the optical depth,

$$
\mathrm{T}^{4}=\frac{3}{4} \mathrm{~T}_{\mathrm{e}}^{4}(\bar{\tau}+\mathrm{q}(\bar{\tau}))
$$

In the above equations, $\mathrm{P}$ represents the pressure, $\mathrm{T}$ the temperature, $\rho$ the density, $g$ the gravity, $h$ the height, $\bar{\kappa}$ the mean absorption coefficient, $\bar{\tau}$ the mean optical depths, and $T_{e}$ the effective temperature. In the present study, we have used an analytical expression by Krishna Swamy (1966),

$$
\mathrm{q}(\tau)=1.39-0.815 \mathrm{e}^{-2.54 \tau}-0.025 \mathrm{e}^{-30 \tau},
$$

which is based upon the observation of limb darkening and the line profiles of the sun (Mitchell 1959). The use of this expression for a wide range of values of the effective temperature $T_{e}$ and the surface gravity $g$ does not guarantee us correct models, but nevertheless might be permitted as providing a first approximation.

In the convective region, the equations are

$$
\begin{gathered}
\pi \mathrm{F}_{\mathrm{rad}}=\frac{16 \sigma \mathrm{T}^{4}}{3 \bar{\kappa} \rho \mathrm{H}} \nabla, \\
\pi \mathrm{F}=\frac{16 \sigma \mathrm{T}^{4}}{3 \bar{\kappa} \rho \mathrm{H}} \nabla_{\mathrm{rad}}, \\
\pi \mathrm{F}_{\mathrm{conv}}=\frac{1}{2} \mathrm{C}_{\mathrm{p}} \rho \overline{\mathrm{v}} \frac{\ell}{\mathrm{H}} \mathrm{T}\left(\nabla-\nabla^{\prime}\right), \\
\overline{\mathrm{v}}^{2}=\frac{\mathrm{g} \ell^{2}}{4 \mathrm{H}}\left(\nabla-\nabla^{\prime}\right), \\
\pi \mathrm{F}=\sigma \mathrm{T}_{\mathrm{e}}^{4}=\pi \mathrm{F}_{\mathrm{conv}}+\pi \mathrm{F}_{\mathrm{rad}}
\end{gathered}
$$

where $\pi \mathrm{F}, \pi \mathrm{F}_{\mathrm{rad}}$, and $\pi \mathrm{F}_{\mathrm{conv}}$ represent the total, the radiative, and the convective flux; respectively, $\sigma$ the Stefan-Boltzmann constant, $\mathrm{H}$ the scale height, $\mathrm{C}_{\mathrm{p}}$ the specific heat for constant pressure per 1 gram, $l$ the mixing length, $\bar{v}$ the average velocity of the turbulent element, and $\nabla$ the logarithmic gradient 
$(\mathrm{d} \ln \mathrm{T} / \mathrm{d} \ln \mathrm{p})$. Among the logarithmic gradients, $\nabla_{\mathrm{rad}}$ is a gradient which would be required if the total flux is carried in the form of radiation, $\nabla$ the average gradient, $\nabla^{\prime}$ the gradient for individual turbulent element, $\nabla_{\text {ad }}$ the adiabatic gradient. A convenient interpolation formula for the efficiency factor $\Gamma$ which can be used both in optically thin and thick cases has been given by Henyey, Vardya, and Bodenheimer (1966):

$$
\Gamma=\frac{\nabla-\nabla^{\prime}}{\nabla^{\prime}-\nabla_{\mathrm{ad}}}=\frac{\mathrm{c}_{\mathrm{p}} \rho \mathrm{T} \overline{\mathrm{v}}}{8 v \mathrm{~T}^{4} \theta},
$$

where

$$
\begin{gathered}
\theta=\frac{\omega}{1+\mathbf{y} \omega^{2}}, \\
\omega=\bar{k} \rho \ell, \\
\mathbf{y}=\frac{3}{4 \pi^{2}} .
\end{gathered}
$$

(For details, see references.) Model atmospheres were constructed with the composition shown in Table 1. References concerning the absorption coefficients are $\mathrm{H}$ : Karzas and Latter (1961), $\mathrm{H}^{-}$: Ohmura and Ohmura (1960) using approximation formulae given by Gingerich (1961), He: Huang (1948), Goldberg (1939), and Ueno (1954), $\mathrm{He}^{+}$: Ueno (1954), $\mathrm{He}^{-}$: McDowell, Williamson, and Myerscough (1966), and electron scattering: e.g. Allen (1962).

\section{ACOUSTIC ENERGY FROM THE CONVECTIVE ZONE}

Quadrupole noise generation from isotropic turbulence has been studied by Lighthill (1952, 1954) and Proudman (1952), and applied to the solar convection zone by Osterbrock (1961). The rate of the acoustic noise generation per unit volume derived by Lighthill (1952) is

$$
\mathrm{j}_{1}=\frac{\alpha \rho \overline{\mathrm{v}}^{8}}{\ell \mathrm{v}_{\mathrm{s}}^{5}}
$$

where $\alpha$ is a numerical constant which depends only weakly upon the form of the spectrum of the turbulence and has the value 38 for the Heisenberg spectrum (Proudman 1952), $l$ is the scale length of the turbulence, and $V_{s}$ is the velocity of sound. The upward flux of energy in acoustic waves is 
Table 1

Elements Used in Calculating Models

\begin{tabular}{|l|c|c|c|}
\hline \multicolumn{1}{|c|}{ Element } & $\begin{array}{c}\text { Relative } \\
\text { Number }\end{array}$ & $\chi_{1}(\mathrm{eV})$ & $\chi_{2}(\mathrm{eV})$ \\
\hline Hydrogen & 1.0 & 13.595 & \\
Helium & 0.15 & 24.581 & 54.403 \\
CNO & $10^{-3}$ & 13.614 & 35.108 \\
Heavy elements & $10^{-4}$ & 7.870 & 16.180 \\
\hline
\end{tabular}

Table 2

Mechanical Energy Flux for Models with $\ell=\mathrm{H}$

\begin{tabular}{|l|c|c|c|c|c|}
\hline $\log \mathrm{g}$ & $\mathrm{T}_{\mathrm{e}}$ & $\log \pi \mathrm{F}_{\mathrm{m}}$ & $\log \mathrm{g}$ & $\mathrm{T}_{\mathrm{e}}$ & $\log \pi \mathrm{F}_{\mathrm{m}}$ \\
\hline \multirow{3}{*}{2.0} & 5000 & 8.05 & 4.0 & 5000 & 7.23 \\
& 6000 & 8.27 & & 6000 & 7.72 \\
& 6500 & 7.72 & & 7000 & 7.94 \\
& 7000 & 2.60 & & 7500 & 7.38 \\
& 8000 & -10 & & 8000 & 4.67 \\
& 5000 & 7.67 & 5.0 & 6000 & 7.36 \\
& 6000 & 8.18 & & 7000 & 7.60 \\
& 6500 & 8.07 & & 8000 & 7.44 \\
& 7000 & 7.47 & & 8500 & 5.91 \\
& 7500 & 4.23 & & & \\
& 8000 & -2.47 & & & \\
\hline
\end{tabular}




$$
\pi \mathrm{F}_{\mathrm{m}}=\frac{1}{2} \int \mathrm{j}_{1} \mathrm{dh}
$$

Table 2 shows the results for models with various values of $T_{e}$ and $g$ when the mixing length is $\ell=H$. As $j_{1}$ is proportional to $v^{8}$, the flux is very much dependent upon the assumed value of the mixing length. Calculation for several cases show that the result should be multiplied by a factor of 10 if we take $\ell=2 \mathrm{H}$. Therefore, the results in Table 2 should be understood as indicating not the absolute value of the acoustic energy flux but the active region in the $T_{e}-g$ plane on a relative scale. Kuperus (1965) has estimated the acoustic energy flux in a very approximate way using the scale height and the maximum velocity given by Böhm-Vitense (1958). Comparisons between his and the present results show that a model calculation, at least, is necessary for a proper discussion of the history of coronae.

\section{STELLAR ROTATION AND MASS LOSS FROM CORONAE}

Brandt (1966) and Weber and Davis (1967) studied the torque exerted by the solar wind upon the sun through its magnetic field. According to them, the characteristic time of the deceleration of the solar rotation is $7 \times 10^{9}$ years, which is comparable to the age of the sun. Mestel (1968) treated the same problem in a more elaborate way but his result was 20 times larger than the ones by Brandt and Weber and Davis. The difference between Mestel and the others may have been caused by the use of different boundary conditions, namely the coronal temperature and density in Mestel's (1968) case, and the solar wind data in Brandt's (1966) and Weber and Davis' (1967) work. The characteristic time is expressed as follows by Weber and Davis (1967):

$$
\frac{1}{\tau}=\frac{2 \mathrm{r}_{\mathrm{a}}^{2}}{3 \mathrm{I}} \frac{\mathrm{dM}}{\mathrm{dt}}
$$

where $I$ is the moment of inertia, $\mathrm{dM} / \mathrm{dt}$ the rate of mass loss, and $r_{a}$ is the distance at which the radial gas velocity $u$ becomes equal to the radial Alfvénic velocity

$$
\frac{4 \pi \rho \mathrm{u}^{2}}{\mathrm{~B}_{\mathrm{r}}^{2}}=1 \quad\left(\text { at } \mathrm{r}=\mathrm{r}_{\mathrm{a}}\right)
$$


Equation (16) indicates that the magnetic field can exert torque to the gas flow up to $r=r_{a}$, therefore the matter which flows along the magnetic line of force gains energy as well as angular momentum until it reaches $r=r_{a}$. In the solar wind, $r_{a}$ lies between 15 and 50 solar radii according to Weber and Davis (1967).

The rate of mass loss is almost independent of the value of $r_{a}$ if it is larger than $r_{c}$, which is the distance where the flow is trans-sonic. In the case of zero rotation (Parker 1958),

$$
\frac{\mathrm{GM} \mathrm{m}_{\mathrm{H}}}{\mathrm{r}_{\mathrm{c}}}=\frac{\mathrm{kT}}{2}
$$

The above implies that the change of the flow from subsonic to supersonic occurs at the distance where the gravitational force becomes so small that it can no longer hold the gas against the expansion caused by the thermal energy. In analogy to equation (18), we may consider that the following relation holds in a rigidly rotating corona;

$$
m_{H}\left(\frac{G M}{r_{c}}-r_{c}^{2} \Omega^{2}\right)=\frac{k T}{2}
$$

Let us define two distances $r_{c, 1}$ and $r_{c, 2}$, where

and

$$
\frac{\mathrm{GM}}{\mathrm{r}_{\mathrm{c}, 1}^{2}}=\mathrm{r}_{\mathrm{c}, 1} \Omega^{2}
$$

$$
\frac{\mathrm{GM} \mathrm{m}_{\mathrm{H}}}{\mathrm{r}_{\mathrm{c}, 2}}=\frac{\mathrm{kT}}{2} \text {. }
$$

Then

$$
r_{c} \sim r_{c, 1}
$$

when $\Omega$ is large (stage $I$ ), and

$$
r_{c} \sim r_{c, 2}
$$

when $\Omega$ is small (stage II). For the coronae of solar-type stars, the transition from stage I to stage $I$ occurs at around $\Omega \sim 2 \times 10^{-5}$, which corresponds to about $15 \mathrm{~km} / \mathrm{sec}$ of rotational velocity at the surface. 
If the critical distance is small, the escape of gas from the gravitational potential occurs where the gas is dense. Therefore we might expect a larger rate of mass loss when a star is rotating faster. The increase in mass loss causes a slight decrease in $r_{a}$, but the product $r_{a}^{2}$ (dM/dt) increases. Thus we may expect a faster decay of stellar rotation when the rotational velocity is greater than $15 \mathrm{~km} / \mathrm{sec}$.

\section{THE EVOLUTIONARY POINT OF VIEW}

In Figure 1, evolutionary tracks of stars from the contraction phase to the main sequence (Iben 1965) have been drawn on the $\mathrm{T}_{e}-\mathrm{g}$ plane, together with a plot of the mechanical energy flux calculated in Section 3.

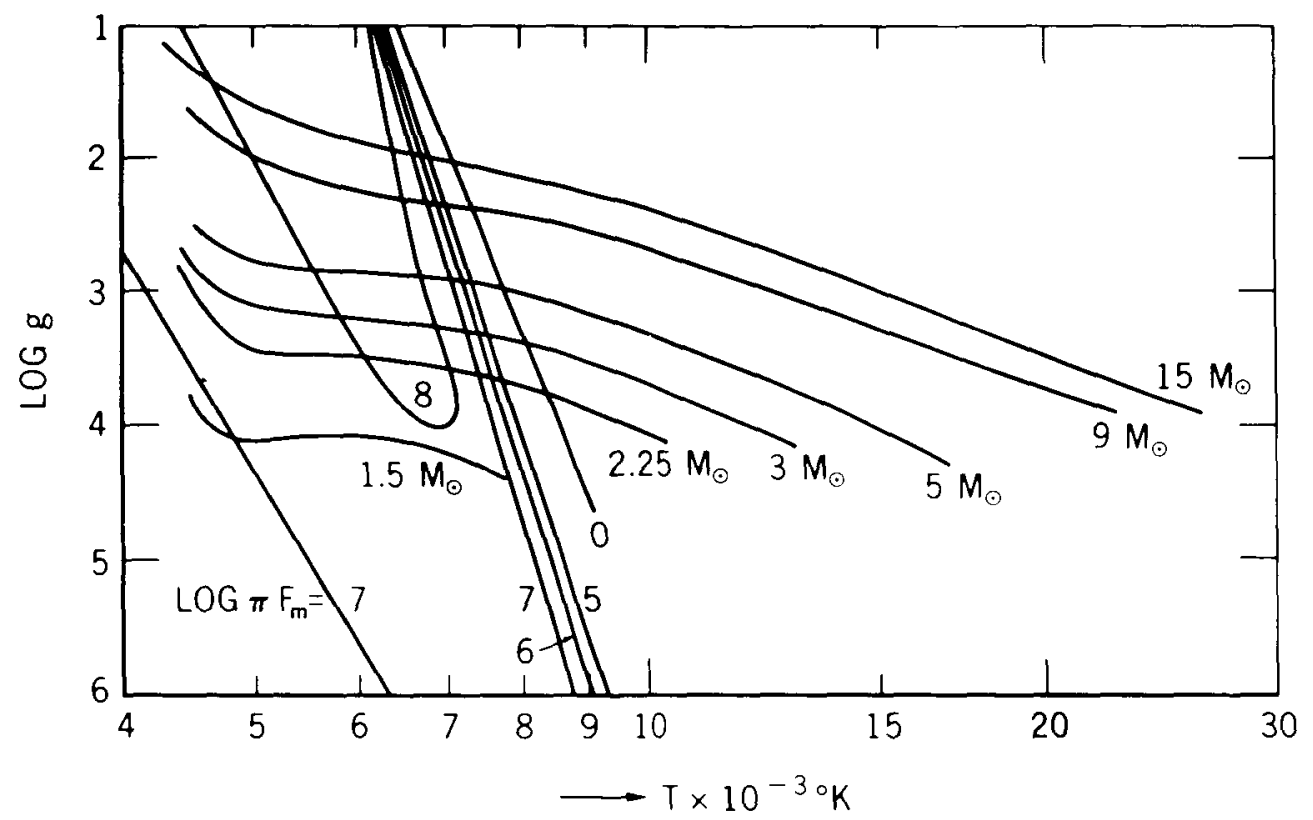

Figure 1-Acoustic Energy Generation Rate and Evolutionary Tracks

Stars with mass $M<1.5 M_{\odot}$ remain in the active region from the contraction to the main sequence phase. From the discussions in the previous section we may conclude that the characteristic time for decelerating the rotation was smaller than the lifetime of the star by a factor of more than, say, 10, when the star was rotating rapidly. This period of fast rotation and large mass loss might also be the period of strong $\mathrm{Ca} I \mathrm{H}-\mathrm{K}$ emission, with strong mass loss changing the chromospheric structure. Then, the above interpretation is in good 
agreement with Wilson's (1966) conclusion that strong H-K emission is found in stars near the zero-age main sequence.

Stars with mass $\mathrm{M}>1.5 \mathrm{M}_{\odot}$ have active coronae only during a part of the contraction phase, which is usually less than $10^{7}$ years. In ordinary cases these stars cannot lose their angular momentum because the time scale is smaller compared to the characteristic time of deceleration. This is in very good correlation with the observational fact that the stellar rotational velocity changes abruptly at spectral type F2, from more than $100 \mathrm{~km} / \mathrm{sec}$ for the hotter stars to less than $20 \mathrm{~km} / \mathrm{sec}$ for the cooler stars within a mass range of $5 \%$ of the mass of a F2 star (Wilson 1966).

T Tauri stars might be interpreted to be stars with $\mathrm{M}>1.5 \mathrm{M}_{\odot}$ passing the active phase. If a strong magnetic field is present as in Ap stars, even massive stars can lose their angular momentum in such a short period. Duplicity helps the mass loss to become greater (Nariai, 1967). Am stars may be interpreted to be massive stars that had strong mass loss and have been decelerated during the contraction phase because of duplicity. ( $T$ Tauri, Ap, and Am stars will be discussed in the next two sections.)

\section{T TAURI STARS}

$\mathrm{T}$ Tauri stars show emission in the hydrogen and $\mathrm{H}-\mathrm{K}$ lines, show displaced absorption line indicating violent mass loss, have large rotational velocities ranging from 20 to $65 \mathrm{~km} / \mathrm{sec}$, have higher luminosities than stars of the same spectral type at the main sequence, and are associated with young clusters whose ages are $\lesssim 10^{7}$ years. (For details, see the review paper by Herbig [1962].) These last two facts indicate that these stars are in the contraction stage.

In the active convection region of contracting stars (see Figure 1), there is usually more acoustic energy generation and less gravitational energy per unit mass at the surface than is to be found in the main sequence stars. These two conditions together with rapid rotation provide favorable conditions for mass loss from the corona. Kuhi's (1964) analysis showing that the more massive stars lose mass at larger rates is consistent with the present interpretation. Kuhi (1964) derived masses from luminosities and colors with the help of Iben's (1965) evolutionary track for the contraction phase. Therefore, "more massive" means "more luminous" here, and can be interpreted as meaning "having less gravitational energy at the surface." The mechanical energy transferred into the corona is spent either in (1) accelerating the flow, or (2) pushing the gas out of the gravitational potential of the star, or (3) causing radiation loss through $\mathrm{X}$-rays, or (4) causing the conduction flow back to the chromosphere. When the 
gravity is sufficiently high, the first two processes are negligible and the temperature of the corona can be obtained by a procedure used by Kuperus (1965) as a function of the acoustic energy generation rate. But when the gravity is not so large, as in the case of $T$ Tauri stars, the temperature does not attain so high a value as Kuperus (1965) suggests, because the energy is consumed in the first two processes. The above discussions are drawn from Uchida (1967) and Unno (1967). What we can expect from the above discussions and the calculations in Section 3 is that more luminous stars that have lesser gravity but have larger acoustic energy generation must show larger mass loss, which is in good agreement with Kuhi's (1964) observation.

\section{PECULIAR A STARS}

The discussions in Section 5 showed that a star with mass $M>1.5 \mathrm{M}_{\odot}$ cannot lose its angular momentum during the $\mathrm{T}$ Tauri phase if the magnetic field is of the order of the solar value (1 gauss at the surface). However, there are stars called peculiar A-type stars because they show peculiar abundance anomalies. These stars have strong magnetic fields ranging from several hundred up to 34 kilogauss in the extreme case. The surface temperatures of these stars correspond to those of normal stars in the spectral range B5 $\sim \mathrm{F} 0$. The implication is that the masses of these stars are greater than $1.5 \mathrm{M}_{\odot}$. The rotational velocities of these stars are remarkably smaller than those of normal stars of the same spectral class. (For details, see Cameron 1967). Since the Alfvén velocity is proportional to the magnetic field intensity, $r_{a}$ is larger in Ap stars than in ordinary stars. Therefore the characteristic time of deceleration of the stellar rotation may become less than the contraction time for Ap stars. As the strong magnetic field and rapid rotation both tend to suppress the turbulent motion, the whole discussion in this section may prove to be invalid when the problem is solved rigorously, because we are applying the rate of acoustic energy generation under conditions of zero (weak) magnetic field to the deceleration of rotation through a stellar wind with a strong magnetic field. Nevertheless, the following comment may be worth mentioning. In the gravitational field, heavier elements tend to concentrate toward the center while lighter elements rise toward the surface, according to the Boltzmann's law. The reason we do not see the separation of elements in the spectrum of stars is that the characteristic time of diffusion is longer than the stellar lifetimes. (See Eddington 1926.) The diffusion time is expressed as

$$
\tau_{\text {diff }}=\frac{x^{2}}{D},
$$


where $\mathrm{x}$ is the characteristic length and $\mathrm{D}$, the diffusion coefficient, is given by

$$
\mathrm{D}=\frac{1}{3} \lambda \mathrm{V}_{\mathrm{s}} \sim 10^{8} \frac{\mathrm{T}^{2 \cdot 5}}{\mathrm{~N}}
$$

where $\lambda$ is the mean free path and $V_{s}$ the velocity of sound. In the corona, however, the characteristic diffusion time is of the order of from a few hours to several days because the density is so low and the temperature is so high. Therefore, if the mixing time is more than the diffusion time, we may expect that heavier elements will be relatively less abundant in the upper corona. Then we may also expect that the same situation will occur in the stellar wind, which is governed by the physical state of the upper corona. Thus, the remaining corona becomes richer in the heavy elements. If we take the particle point of view in interpreting the stellar wind, the situation becomes more extreme. In this interpretation, the stellar wind is composed of the particles which do not collide with other particles since they have gained escape velocity. Then, as the heavier elements have smaller thermal velocities, the height at which the escape occurs effectively for heavier elements is higher than that for lighter elements. Since larger height implies smaller density, the enrichment of heavier particles in the remaining corona occurs even without element separation caused by the gravitational field.

Keeping this fact in mind, let us proceed now to the next part of the discussion. Figures 2 and 3 show the acoustic energy generation rate and the thickness of the convection zone in $\mathrm{g} \mathrm{cm}^{-2}$ as a function of time, respectively, for a star with $2.25 \mathrm{M}_{\odot}$. A gap in the thickness at $1.75 \times 10^{6}$ years is due to the separation of the convection zone into two parts, the upper one being due to hydrogen ionization and the lower one to helium II ionization. Acoustic energy generated after this separation is $1.5 \times 10^{21} \mathrm{erg} \mathrm{cm}^{-2}$. A part of this energy is spent in pushing the material out of the gravitational potential of the star. The ratio of the energy required for pumping the matter out and the energy generated in the convection zone is about $10^{-2}$ for the sun. This factor might be larger for stars with mass $\mathrm{M}>1.5 \mathrm{M}_{\odot}$ at $\mathrm{T}$ Tauri stage because these stars have smaller values of $\mathrm{g}$. Stronger magnetic fields in Ap stars also assist the transfer of energy into the corona without its being consumed in the chromosphere. If we assume 0.1 for that ratio, then the total amount of material which passes through the convection zone is about $10^{3}$ times larger than the material contained in the convection zone. Thus we can expect enrichment of heavier elements in the visible thin layer in stars with mass $M>1.5 \mathrm{M}_{\odot}$. The diffusion time at the bottom of this concentrated region is of the order of $10^{12}$ years. Therefore, unless a mechanism other than diffusion is at work, we would observe metal-rich stellar surfaces. Then the spectral difference between Ap and normal stars could be 


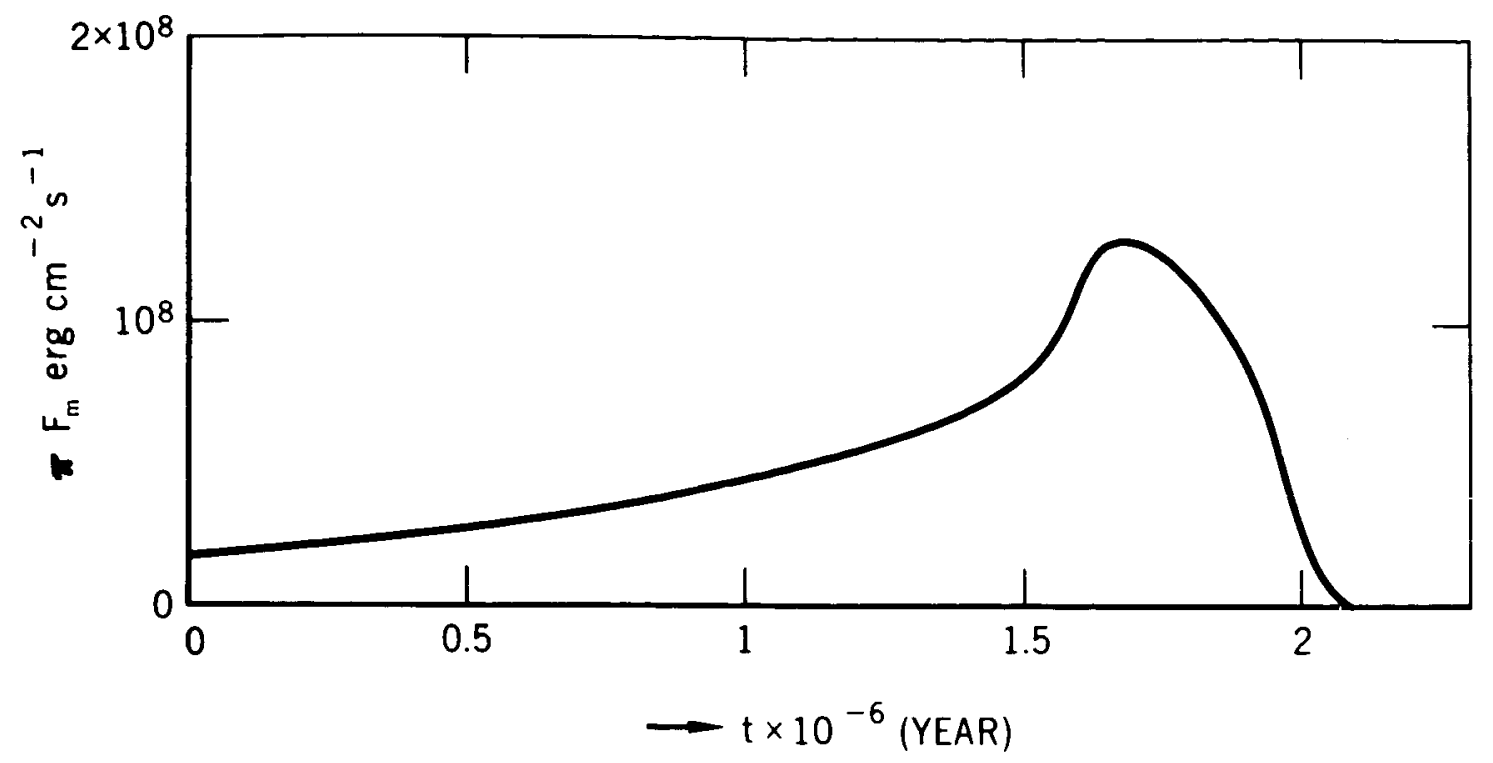

Figure 2-Change of the Mechanical Energy Flux with Time for $M=2.25 M_{\odot}$

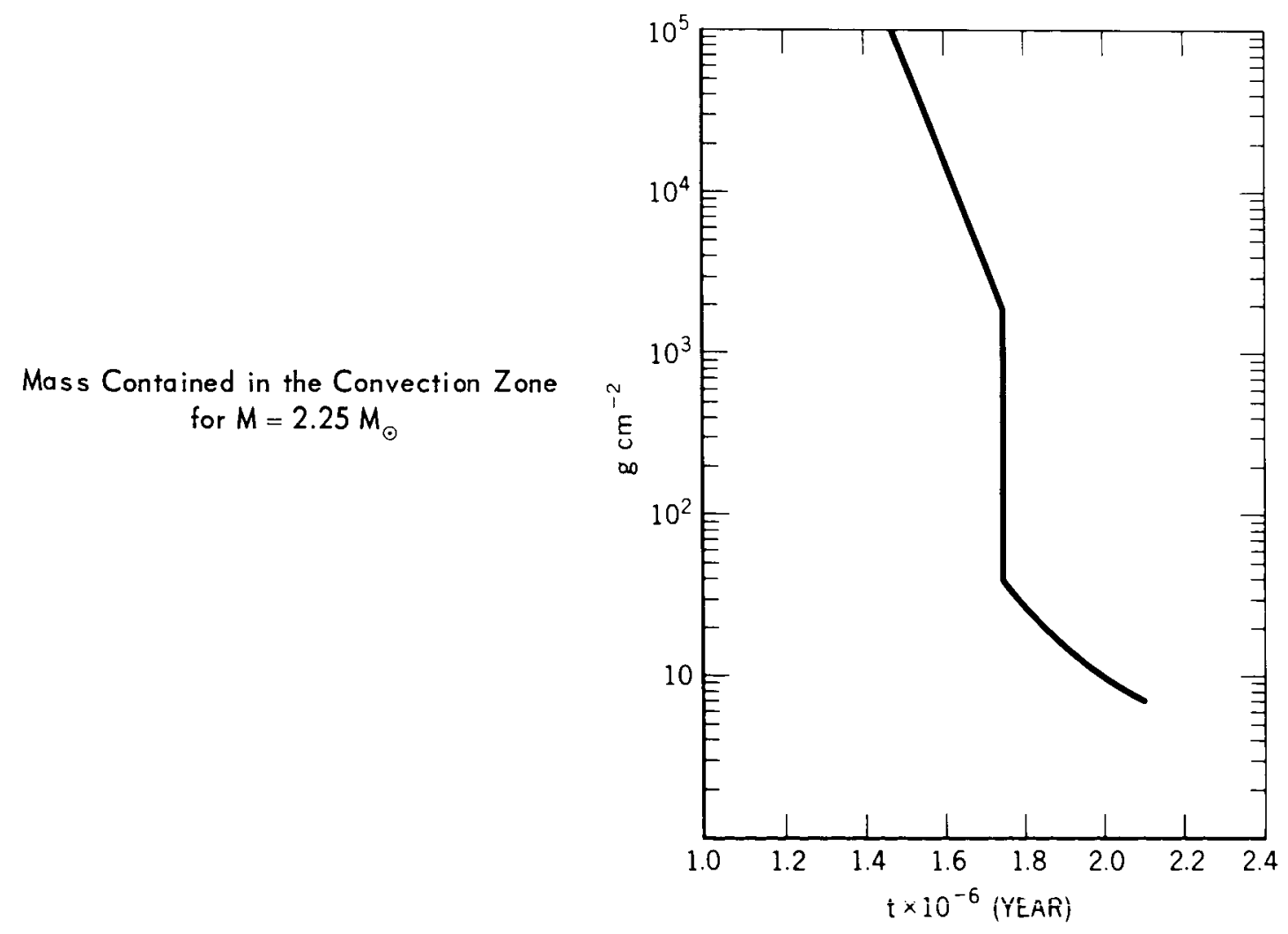


interpreted as due to (1) the small circulatory motion because of slow rotation, of Ap stars and (2) the suppression of the circulatory motion by the magnetic fields in Ap stars.

Almost the same discussion can be applied to Am stars. The difference between Ap and Am cases is that the existence of a companion may raise the mass loss rate from coronae of Am stars, which may cause the differences in the degrees of concentration of metals.

\section{ACKNOWLEDGMENT}

The author's thanks are due to Dr. R. C. Cameron and Dr. M. P. Nakada and many other colleagues of the Goddard Space Flight Center for useful discussion and encouragement. The author holds an NRC-NASA Resident Research Associateship.

This paper was presented at the Second Trieste Colloquium on "Mass Loss from Stars," held from September 12-14, 1968, in Trieste, Italy. 


\section{REFERENCES}

Allen, C. W.: 1962, Astrophysical Quantities, The Athlone Press.

Biermann, L.: 1948, Z. Astrophysik 25, 161.

Böhm-Vitense, E.: 1958, Z. Astrophysik 46, 108.

Brandt, J. C.: 1966, Astrophys. J. 144, 1221.

Cameron, R. C., editor: 1967, The Magnetic and Related Stars, Mono Books Corp., Baltimore.

Eddington, A. S.: 1926, The Internal Constitution of Stars, Cambr. Univ. Press.

Gingerich, O.: 1961, Astrophys. J. 134, 653.

Goldberg, L.: 1939, Astrophys. J. 90, 414.

Henyey, L., Vardya, M. S., and Bodenheimer, P.: 1965, Astrophys. J. 142, 841.

Herbig, G.: 1962, Advances in Astronomy and Astrophysics, 1, 47.

Huang, S-S.: 1948, Astrophys. J. 108, 354.

Iben, I.: 1965, Astrophys. J. 141, 993.

Karzas, W. J. and Latter, R.: 1961, Astrophys. J. Suppl. $\underline{6}, 167$.

Kuhi, L. V.: 1964, Astrophys. J. 140, 1409.

Kuperus, M.: 1965, Recherches Astronomique de l'Obs. d'Utrecht, XVII (I).

Lighthill, M. J.: 1952, Proc. Roy Soc. A211, 564.

Lighthi11, M. J.: 1954, Proc. Roy. Soc A222, 1.

McDowell, M. R. C., Williamson, J. H., and Myerscough, V. P.: 1966, Astrophys. J. 144,831 .

Mestel, L.: 1968, Monthly Not. Roy. Astron. Soc. 138, 359.

Mitchell, W. E.: 1959, Astrophys. J. 129, 369. 
Nariai, K.: 1967, Publ. Astron. Soc. Japan 19, 564.

Ohmura, T. and Ohmura, H.: 1960, Astrophys. J. 131, 8.

Osterbrock, D. E.: 1961, Astrophys. J. 134, 347.

Proudman, I.: 1952, Proc. Roy. Soc. A214, 119.

Schatzman, E.: 1949, Annales d'Astrophys. 12, 203.

Schwarzschild, M.: 1948, Astrophys. J. 107, 1.

Spiegel, E. A.: 1957, Astrophys. J. 126, 202.

Swamy, K. S. Krishna: 1966, Astrophys. J. 145, 174.

Uchida, Y.: 1967, Proc. Tokyo Symp. UV and IR Radiation from Celestial Bodies (in Japanese) p. 60.

Ueno, S.: 1954, Contr. Inst. Astrophys. Univ. Kyoto, No. 42.

Unno, W.: 1967, Proc. Tokyo Symp. UV and IR Radiation from Celestial Bodies (in Japanese) p. 71.

Weber, E. J. and Davis, L. Jr.: 1967, Astrophys. J. 148, 217.

Wilson, O. C.: 1966, Astrophys. J. 144, 695. 